\title{
Evaluation of the Systemic Response to Boiling Histotripsy Treatment for Renal Carcinoma George R. Schade, MD
}

Final Report May 1, 2017

Boiling histotripsy (BH) is an experimental non-invasive focused ultrasound (FUS) technology distinct from cavitation cloud histotripsy. BH uses milliseconds-long FUS pulses at low-duty cycle to non-thermally mechanically homogenize tissue. We have been developing $\mathrm{BH}$ as a non-invasive treatment for renal carcinoma (RCC). This proposal aimed to evaluate the local tumor effects of $\mathrm{BH}$ ablation over time and characterize the systemic immune response to $\mathrm{BH}$ ablation of RCC using the Eker rat de novo RCC model.

To accomplish these aims, RCC bearing genotyped Eker rats (Tsc2 heterozygotes) with tumors $>5 \mathrm{~mm}$ and syngeneic non-tumor bearing WT rats were randomly assigned to transcutaneous BH or an FUS SHAM procedure targeting $\sim 0.5 \mathrm{cc}$ of RCC or non-tumor bearing normal kidney. BH was delivered with a $1.5 \mathrm{MHz}$ US-guided small animal FUS system (VIFU-2000, Alpinion) operated at duty cycles of 1-2\% with 10-20 ms pulses and 525-600 W electric power. Immediately following treatment and on "postoperative" (POD) 1 rats received normal saline $10 \mathrm{mg} / \mathrm{kg} \mathrm{SQ}$ and ketoprofen $5 \mathrm{mg} / \mathrm{kg} \mathrm{SQ}$ for analgesia. They were then recovered from anesthesia and monitored for pain and complications until the time of euthanasia on POD 7, 14, or 56 . To monitor the treated tumor, rats underwent serial US imaging surveillance under anesthesia POD 1, 2, 7, 14, 28, and 56 . Following euthanasia, the treated and contralateral kidney, tumor draining lymph nodes (TDLN), and spleen were collected. The kidneys were formalin fixed and then processed for histologic assessment with hematoxylin and eosin staining to assess local effects of $\mathrm{BH}$ treatment and healing of the treated volume. Additionally, the kidneys were processed for immunohistochemistry to evaluate the infiltrating leukocyte populations following $\mathrm{BH}$ and sham procedures. TDLNs and spleens were processed for Flow-cytometry (LSRII) to evaluate for changes in leukocyte populations in lymphoid organs following treatments

As outlined in our previous progress reports, several of our Eker rats required humane euthanasia from sequelae of their Tsc2 carrier status prior to undergoing $\mathrm{BH} /$ sham treatment. This required adjustments to our allocation arms. In total 31 WT rats were treated $(n=12$ sham, $n=19 \mathrm{BH})$ and 38 Eker rats were treated $(n=12, n=26 \mathrm{BH})$. Following treatment rats were survived for 7,14 , or 56 days. No rats required additional ketoprofen beyond POD1.

\section{Evolution of Treatment}

As expected, $\mathrm{BH}$ treatment of both normal kidney and RCC produced a heterogeneous hypoechoic cavity seen on B-mode US (Figure 1). Over the first 7 days post-treatment the cavity became increasingly hypoechoic, peaking on POD7 with a nearly anechoic well defined cavity. Subsequently, the cavity shrank and became barely apparent by POD28 and was no longer apparent in any rat by POD56. Histologically, good correlation was seen with US findings. On POD7, the targeted volume of kidney/RCC was visible as a cavity of homogenized tissue devoid of cellular features with sharp borders between treated and untreated tumor (Figure 2). On POD14, the cavity a band of fibrosis around

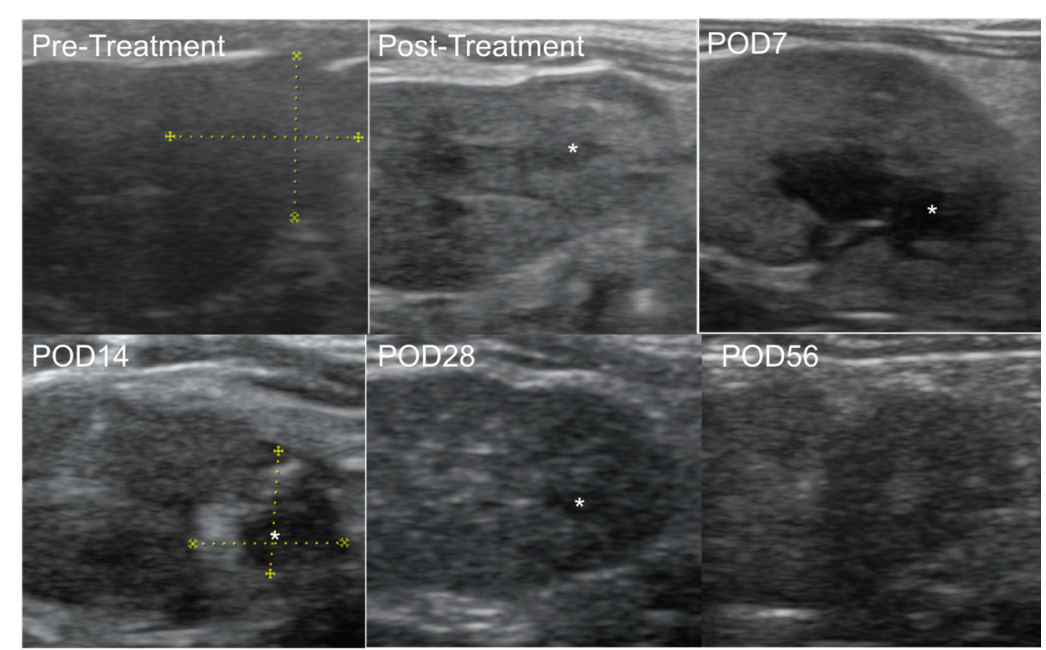

Figure 1: US appearance of the evolution of $\mathrm{BH}$ treated RCC over time. Immediately following treatment, $\mathrm{BH}$ produced a mildly hypoechoic cavity $\left({ }^{*}\right)$ that became increasingly hypoechoic and well defined through POD7. By POD14 the cavity was less hypoechoic and smaller, was barely visible by POD28, and no longer apparent on POD56. 


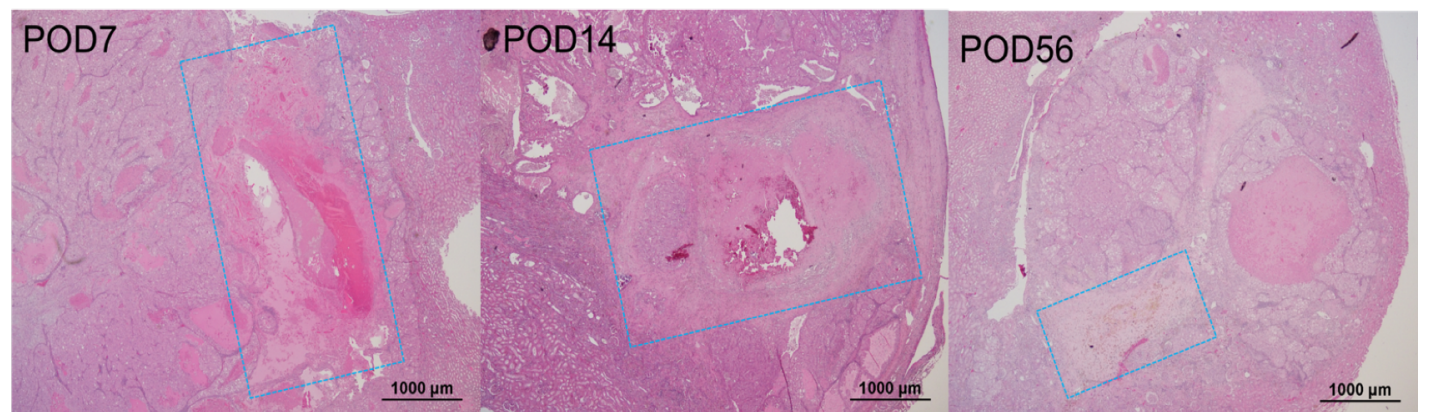

Figure 2: Histologic appearance of $\mathrm{BH}$ treated RCC over time. On POD7, the treatment volume was visible as a cavity of homogenized tissue (blue box) devoid of cellular features with sharp borders between treated and untreated tumor. On POD14 the cavity contained less homogenized tissue and had a band of fibrosis at the periphery suggesting resorption and contraction. On POD56 the treated tumor was completely resorbed leaving behind a small fibrotic scar with contraction of the untreated portion of the tumor around the treatment site. the treatment cavity was apparent suggesting resorption of the homogenized tissue and contraction of the treated volume. On POD56, the treatment site within kidney/tumor was completely resorbed leaving behind a fibrotic scar containing dystrophic calcifications.

normal kidney, this was associated with contraction of the untreated parenchyma around the scar and an apparent dimple on the surface of the kidney or "absence" of the lower or upper pole of the kidney when targeting the renal poles. Within tumors, a similar fibrotic scar was apparent and the untreated portion of

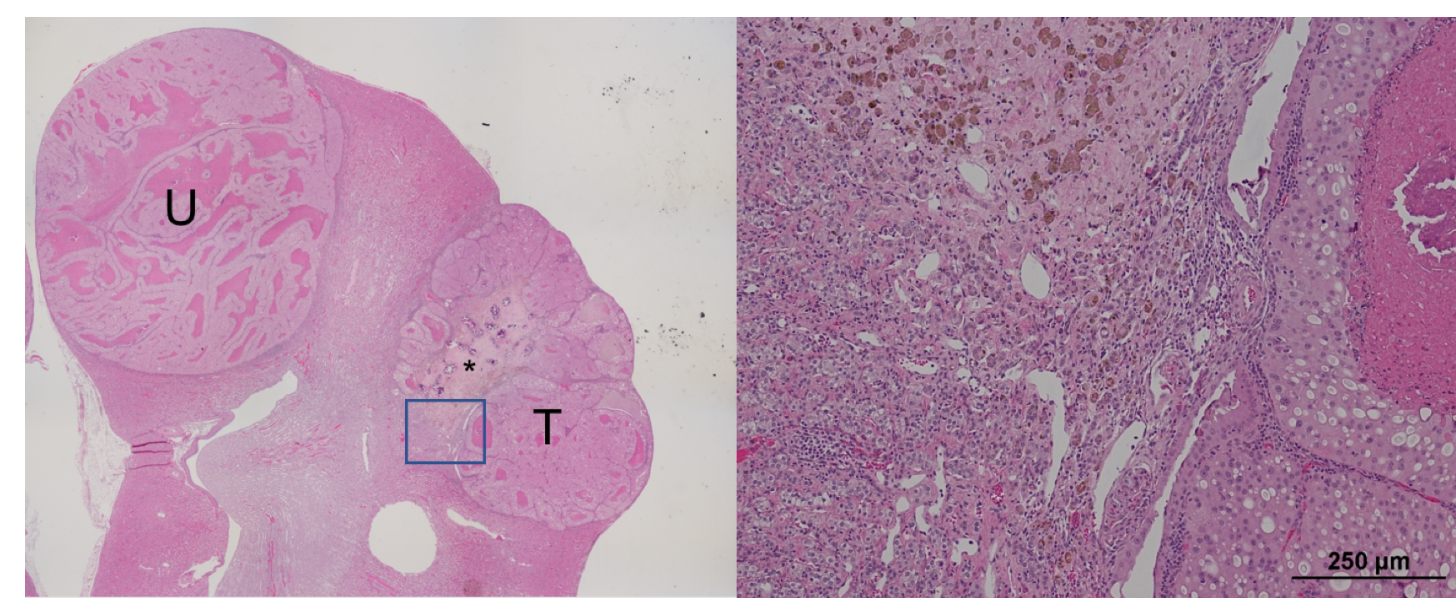

Figure 3: Histologic appearance of untreated $(\mathrm{U})$ and $\mathrm{BH}$ treated $(\mathrm{T}) \mathrm{RCC}$ after 56 days, demonstrating contraction of the (now) irregularly shaped BH treated tumor around a central scar $\left(^{*}\right)$ with areas of

the targeted tumor appeared irregularly shaped (as opposed to spherical or ovoid) (Figure 3) and slightly fibrotic consistent with the tumor shrinking around the ablated tumor volume.

Based on US and histologic evidence of tumor shrinkage following $\mathrm{BH}$, we analyzed the 4-week change in $\mathrm{BH}$ treated tumor volume among Eker rats survived to POD56. Among these rats, BH produced a significant decrease in tumor volume from $222.7 \pm 49.0 \mathrm{~mm}^{3}$ pretreatment to $131.2+/-42.1 \mathrm{~mm} 3$ on POD28 ( $p=0.03$ ). Observed mean change in tumor volume $\left(-91.5 \pm 24.2 \mathrm{~mm}^{3}\right)$ was similar to expected $\left(-112.8 \pm 22.1 \mathrm{~mm}^{3}\right)$ $(p=0.11)$.

\section{Local Immune Response to Boiling Histotripsy}

Following $\mathrm{BH}$ ablation of both normal kidney (WT rats) and RCC (Eker rats) an immune infiltration was seen surrounding the ablated volume of tissue at POD7 and POD14. In WT rats, on POD56 the kidney appeared normal aside from the scar in $6 / 7$ with the remaining rat having significant pan-kidney inflammation of unclear significance. Conversely, in all $n=8$ Eker rats, the treated RCC demonstrated residual immune infiltrate (Figure 3) surrounding the scar. Overall, the immune
Treated Tumor CD8+ CD62L- CD44+ T-lymphocytes

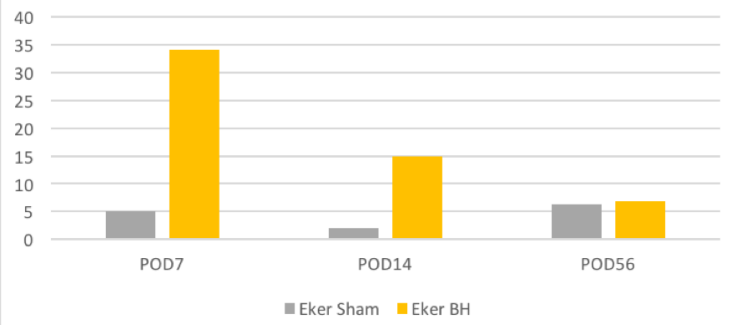

Figure 4: Flow cytometry results evaluating leukocyte populations within the treated tumor of Eker rats stratified by treatment (Sham vs $\mathrm{BH}$ ) over time. 
infiltration into $\mathrm{BH}$ treated RCCs was qualitatively similar to slightly increased compared sham treated tumors at POD56.

To characterize the infiltration seen in both $\mathrm{BH}$ treated and sham treated WT kidneys and Eker RCC/kidneys we attempted to perform IHC targeting T-cells (CD8, CD4, CD 44, FoxP3), antigen presenting cells (CD11C, F4/80, $\mathrm{MHCII}$ ) and the program cell-death pathway (PD-1, PDL-1). However, despite attempting different antibodies and multiple staining conditions in conjunction with the UW histology core we could not get reliable staining. Consequently, in the last $\sim n=2$ of each Eker treatment group BH vs. Sham for POD7, POD14, and POD56), a piece of the treated tumor was processed for flowcytometry. Due to the small number of rats in each group statistical analysis was not performed, however, graphically $\mathrm{BH}$ may be associated with increased infiltration of CD8+ CD62L- CD44+ effector memory T-cells $\left(T_{e m}\right)$ in to the targeted tumor on POD7 and POD14 (Figure 4) with similar distribution between $\mathrm{BH}$ and sham on POD56. No patterns were observed for CD4+ T-cell populations, $\mathrm{CD} 11 \mathrm{c}+$ dendritic cells, or $\mathrm{F} 4 / 80+$ macrophages.

\section{Systemic Immune Response to Boiling Histotripsy}

In addition to assessing the local immune response we evaluated for changes in the immune system systemically performing flow

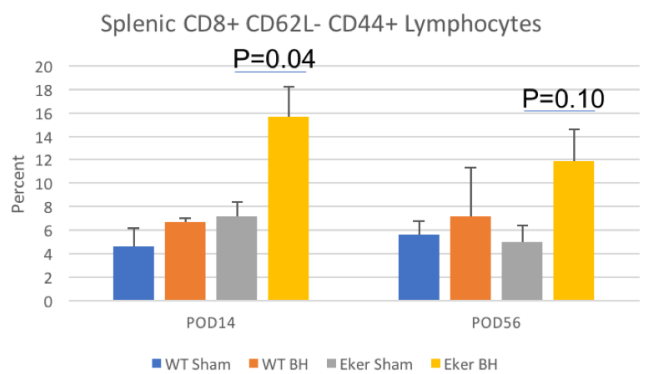

Figure 5: Flow cytometry results evaluating leukocyte populations within spleens stratified by rat type (WT vs Eker) and treatment (Sham vs BH) 14 and 56 days post-treatment demonstrating significantly elevated CD8+ CD62L- CD44+ effector/memory T-cells on POD14 and near significant elevations on POD56 in $\mathrm{BH}$ vs sham treated Eker rat RCC, without significant changes in WT rats. No significant differences were observed in CD4+ T-cells, T-

cytometry to characterize leukocyte populations in the spleen and TDLNs. Flow cytometry antibodies anti CD3, CD4, CD8, Foxp3, CD62L, CD44, CD11C, MHCII, and F4/80. In the spleen, BH treatment of RCC in Eker rats, but not of normal kidney in WT rats, was associated with a significant $\sim 2$-fold increase in CD8+ Tem lymphocytes vs. Sham on POD14 ( $p=0.04$, Figure 5). On POD56, this effect was similar in magnitude but missed statistical significance $(p=0.10)$. There were no other significant differences observed in the spleen between $\mathrm{BH}$ and sham treated animals at any time point for T-cells (including CD8+. CD4+, and Tregs), dendritic cell, or macrophage populations.

Within TDLNs, however, significant changes were observed in CD4+ and CD8+ $\mathrm{t}$-cell populations that were specific to $\mathrm{BH}$ ablation of RCC. Specifically, BH ablation of RCC, but not normal WT kidney, produced significantly increased $(\sim 1.2 \quad$ Fold $)$ populations of CD4+ CD62LCD44+ Tem cells in TDLNs on POD7 and POD14 (Figure 6) that did not persist to
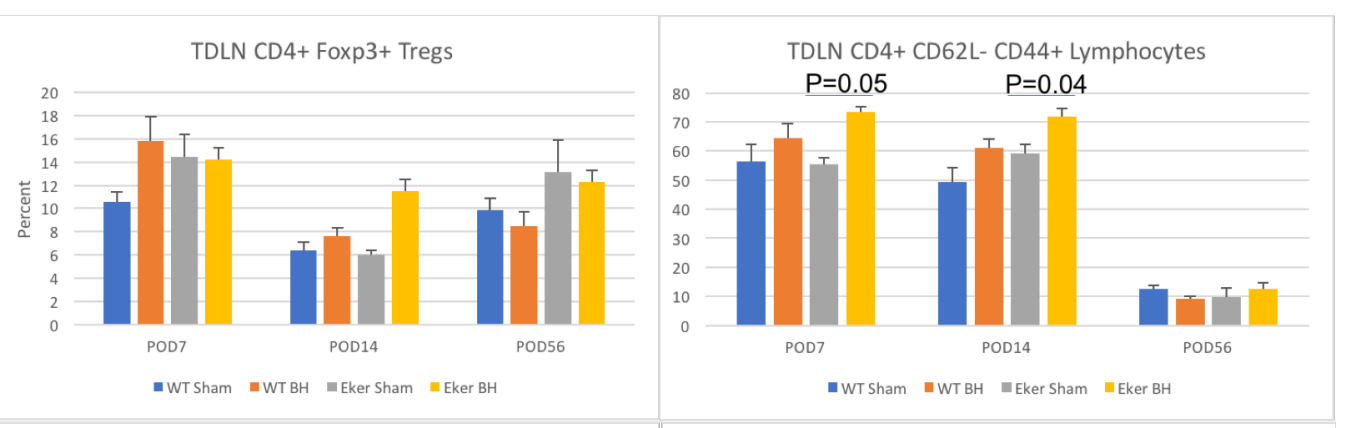

TDLN CD8+ CD62L- CD44+ Lymphocytes

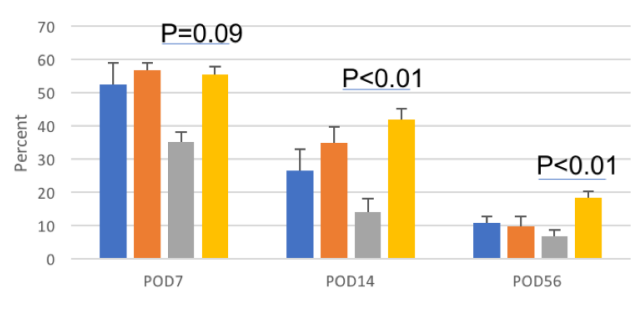

-WT Sham $\quad$ =WT BH $=$ Eker Sham $\quad$ EEker BH
$\mathrm{CD} 8+\mathrm{CD} 62 \mathrm{~L}+\mathrm{CD} 44+$ Lymphocytes

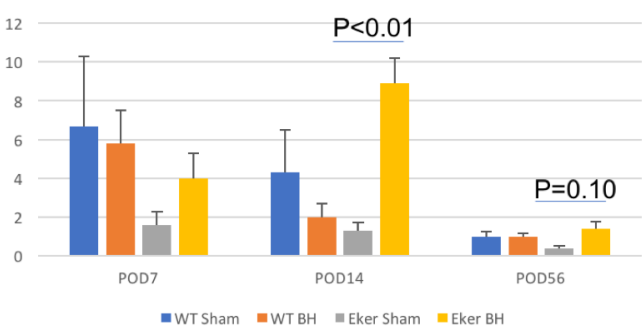

Figure 6: Flow cytometry results evaluating leukocyte populations within tumor draining lymph nodes (TDLNs) stratified by rat type (WT vs Eker) and treatment (Sham vs BH) 7, 14, and 56 days post-treatment. BH treatment of RCC in Eker rats, but not WT normal kidneys, was associated

POD56. Among CD8+ T-cells, similar to spleen (and possibly tumor) BH treatment of RCC, but not WT normal 
kidney produced near significant increases in CD 8+ Tem on POD7, with significant increases seen on POD14 that persisted to POD56. Similarly, BH of RCC, but not WT normal kidney, produced significant increases in CD8+ CD62L+ CD44+ central memory (Tcm) T-cells within TDLNs on POD14 with similar near significant persistent increases on POD56. Interestingly for both these CD8+ T-cell populations, Sham treated Eker rats appear to exhibit "suppression" of these cells vs. WT animals, and BH treatment of RCC returns them to "normal" WT levels. Finally, in our previous progress report, there was a near significant increase in CD4+ Tregs on POD14 within TDLNs following RCC BH ablation, however, after completing all rats no differences were observed in Treg populations at any time.

\section{Conclusions}

$\mathrm{BH}$ partial ablation of normal kidney and RCC produced reproducible tissue effects that universally appeared to heal within 8 weeks of treatment. Further, treatment produced tumor volume loss near expected volume loss within 4 weeks of treatment. Finally, BH treatment of RCC, but not WT normal kidney, resulted in measurable changes in systemic (and possible local) T-cell populations. Further work will aim to further characterize the changes in the immune system in response to treatment and understand the mechanism by which immunomodulation occurs.

\section{Presentations:}

Schade GR. Boiling Histotripsy for RCC: Preclinical Development. Invited Plenary Presentation. Engineering and Urology Society, Annual Meeting, May 2016, San Diego, CA.

Schade GR, Wang YN, D'Andrea S, Hwang JH, Lin DW, Bailey MR, Khokhlova TD

Pilot assessment of boiling histotripsy ablation of renal carcinoma in the Eker rat:

feasibility and the response to treatment. Urology Care Foundation Research Honors Program, May 2016. San Diego, CA.

Schade GR, Wang YN, D’Andrea S, Hwang JH, Lin DW, Bailey MR, Khokhlova TD Pilot assessment of boiling histotripsy ablation of renal carcinoma in the Eker rat:

feasibility and the response to treatment. Urology Care Foundation Early Career Investigators Showcase. San Diego, CA.

Schade GR, Khokhlova TD, Wang YN, Star F, Maxwell AD, Kreider W, Bailey MR, Khokhlova V. Pilot Assessment of Transcutaneous Boiling Histotripsy Ablation of the Kidney in the Porcine Model. American Urologic Association, Podium Presentation, Annual Meeting 2016. May 2016, San Diego, CA.

Schade GR, Khokhlova TD, Wang YN, May PC, Maxwell AD, Lin DW, Bailey MR, Khokhlova V. Initial assessment of boiling histotripsy ablation of ex vivo human renal tumors vs. renal cortex. Engineering and Urology Society, Poster Presentation, Annual Meeting 2016. San Diego, CA.

Schade GR, Wang YN, Gravell K, Pillarisetty V, Hwang JH, Liles WC, Khokhlova V, Bailey MR, Khokhlova TD. Characterizing the immune response to boiling histotripsy ablation of renal carcinoma in the Eker rat. Focused Ultrasound Symposium, Podium Presentation, Biennial Meeting, 2016

Schade GR, Wang YN, Gravell K, Pillarisetty V, Hwang JH, Liles WC, Khokhlova V, Bailey MR, Khokhlova TD. Healing and the immune response following boiling histotripsy ablation of renal carcinoma in the Eker rat. Acoustics Society of America, Invited Podium Presentation, Fall Meeting 2016 
Brisbane W, Khokhlova TD, Whang S, Gravell K, Wang YN, Pillarisetty V, Hwang JH, Liles WC, Khokhlova V, Bailey MR, Schade GR. Boiling Histotripsy Ablation Of Eker Rat Renal Carcinoma Produces Significant Changes In the Immune System. Engineering in Urology Society, Annual Meeting, May 2017

Brisbane W, Khokhlova TD, Whang S, Gravell K, Wang YN, Hwang JH, Khokhlova V, Schade GR. Boiling Histotripsy Ablation of Renal Carcinomaln A Chronic Rat Model. American Urologic Association, Annual Meeting May 2017

Schade GR, Brisbane W, Whang S, Gravell K, Wang YN, Pillarisetty V, Hwang JH, Liles WC, Khokhlova V, Bailey MR, Khokhlova TD. Boiling histotripsy ablation of renal carcinoma in the Eker rat produces significant changes in the immune system. International Society of Therapeutic Ultrasound, Annual Meeting, June 2017

\section{Follow-on Funding:}

Grant Title: Focal Therapy of Prostate Cancer using Non-Thermal Tumor Ablation with Focused Ultrasound PIs: Schade GR (US); Khokhlova VA (Russia)

Grantor: $\mathrm{NCl}$

Funding Mechanism: U.S.-Russia Bilateral Collaborative Research Partnerships on Cancer (R21)

Proposal Submitted: 10/28/2016 